UDC: 330.47:336.742::364.3:314.04

DOI: $10.26697 /$ ijes.2019.2.40

\section{Cryptocurrency Possibilities in Target Financing of Public Social Payments}

Professor Melnyk Yu. B. ${ }^{1,2,3}$

${ }^{1}$ National Academy of the National Guard of Ukraine, Ukraine

${ }^{2}$ Kharkiv Regional Public Organization "Culture of Health"(KRPOCH), Ukraine

${ }^{3}$ Scientific Research Institute KRPOCH, Ukraine

\begin{abstract}
Background:

Development of IT-technologies has led to changes in financial systems of different countries promoting their improvement and progress. New financial institutions are being formed as well as new technologies, instruments and forms of financial interaction between people are being developed.

It has become necessary to develop the modern calculating instrument that has resulted in alternative money turnover, which has been called "cryptocurrency". Despite certain problems connected with the cryptocurrency turnover (Bitcoin, Ethereum, Ripple, Litecoin etc.) - its sanctioning by public institutions of the state power, it is widely used as a calculating instrument of additional money markers that are on the same level with legal forms of currencies.

Implementation of a cryptocurrency reveals possibilities which were impossible before for traditional (fiducial) currencies and non-cash payments, in particular controlling transactions as for target use of funds.

The aim of the research is to ground the possibilities of a cryptocurrency in target financing; develop a system model of target financing of social payments to the public by means of cryptocurrencies.
\end{abstract}

\footnotetext{
Methods:

A set of methods has been used in the research: retrospective analysis of implementing a cryptocurrency worldwide; generalising the experience of using cryptocurrency by European countries for public social payments; modelling the financial system (with the use of cryptocurrency) as for public social payments.
}

\footnotetext{
Results:

Acknowledgement of a cryptocurrency by public institutions in countries with developed economy, its popularity at the majority of stock-markets and currency exchange platforms have promoted its approval as a legal means of payment.

Development of the financial market in this direction reveals wide perspectives for implementing a cryptocurrency in transactions connected with target financing on the whole and social payments in particular.

The author has developed a system model of target financing of social payments to the public that
}

presupposes providing various types of social aid and subsidies by the state. Cryptocurrencies with a clear list of possibilities to expend this finance are used as a payment means.

The efficiency of this model is evident as it does not only allow avoiding keeping a staff that controls expenditures of target funds but also makes us sure that these funds will be used for their specific tasks.

Let us draw some examples which in our opinion can be used by government institutions, charity organisations and funds, which provide social aid to the public. Among them are such payments: unemployment benefits; disability pension; maternity grants, birth grants, benefits for single mothers, allowance for looking after a sick child etc.

\section{Conclusions:}

New IT-technologies in the economic sphere have resulted in development of the world financial market and implementation of new currency systems such as a cryptocurrency. The use of a cryptocurrency in transactions connected with target financing of social payments makes this process efficient and controlled.

A perspective direction of the research is to solve legal issues in the sphere of providing social aid with the help of a cryptocurrency as a legal payment means, as well as to develop technological support for this activity.

Information about the author:

Melnyk Yuriy Borysovych - Doctor of Philosophy in Pedagogy, Associate Professor, Professor of the Department of Psychology and Pedagogy, National Academy of the National Guard of Ukraine; Founder and Chairman of the Board, KRPOCH; Director, Scientific Research Institute KRPOCH, Kharkiv, Ukraine.

Research interests: methodology and theory of science, social sciences, education, psychological technology, health culture; https://orcid.org/0000-0002-8527-4638.

Corresponding Author:

Melnyk Yuriy Borysovych

Corresponding Author's Email:

YBM.office@gmail.com 